\title{
Lack of biologically active estrogens in commercial cow milk
}

\author{
C. Furnari, D. Maroun, S. Gyawali, B. W. Snyder, and A. M. Davis ${ }^{1}$ \\ Biology Department, Framingham State University, Framingham, MA 01701
}

\begin{abstract}
Recently, many studies have investigated potential estrogenic compounds in the human diet. Several of these investigations have studied cow milk, a mainstay of the diets of both young and old. In vitro studies have determined that estrogens can be found in milk, and that the concentration of estrogen may be correlated to the fat content in the milk. Regardless, the majority of these studies have concluded that the levels of estrogens in milk are too low to have a physiological effect. However, a recent study found that commercial $1 \%$ cow milk was uterotrophic in rats, suggesting that it contained biologically significant levels of estrogen. Using the rat model, we tested milk samples from commercial sources and with varying fat content for estrogenic activity. Ovariectomized female rats were given milk ad libitum for a period of 2 wk. After $12 \mathrm{~d}$ of treatment, rats were tested sequentially in an open field and an elevated plus maze to determine any effect of milk on anxiety levels. Upon completion of the behavior testing, uterine weights were examined. Regardless of milk type, no difference was observed in daily volume of milk consumed. Contrary to previous publications, no differences existed in either the behavior or the uterine weights between animals that consumed any milk type and the negative controls. These results demonstrated that none of the commercial milk types that we tested contained biologically significant estrogenic activity.
\end{abstract}

Key words: uterotrophic, open field, elevated plus maze, rat

\section{INTRODUCTION}

The milk production of cows that are pasture fed has been found to decrease significantly during the latter half of pregnancy (Gowen, 1924; Erb et al., 1952). Therefore, manufacturers have developed cow feeds containing large amounts of protein that supply the nutrition necessary to increase the amount of milk produced by cows throughout most of their pregnancy

Received March 15, 2011.

Accepted September 5, 2011.

${ }^{1}$ Corresponding author: adavis@framingham.edu
(Cunningham et al., 1996; Komaragiri and Erdman, 1997). This includes the latter half of pregnancy, which is when the concentration of estrogens is the highest in pregnant mammals (Erb et al., 1977; Heap and Hamon, 1979; Pape-Zambito et al., 2008). It is believed that significant amounts of estrogens are still present in cow milk after commercial processing for human consumption is completed (Heap and Hamon, 1979; Ganmaa et al., 2004). If correct, this would create concern for the potential estrogenic effects the milk may have on its consumers.

Published reports vary greatly in the amount of estrogens found in milk. Levels of estradiol (a form of endogenous estrogen) for unprocessed milk range from 1.4 to $85 \mathrm{pg} / \mathrm{mL}$ (Monk et al., 1975; Wolford and Argoudelis, 1979; Pape-Zambito et al., 2007). This range is further complicated by whether the milk is whole or skim, and whether the cow was pregnant. A recent study by Pape-Zambito et al. (2007) demonstrated that $17 \beta$-estradiol was found in greater concentrations in milk from pregnant cows (average of $3.0 \mathrm{pg} / \mathrm{mL}$ ) than in milk from nonpregnant cows (average of 1.3 $\mathrm{pg} / \mathrm{mL}$ ). However, the range of estradiol in the milk samples in their study was from undetectable to as high as $22.9 \mathrm{pg} / \mathrm{mL}$ (Pape-Zambito et al., 2007). Although estrogens are important to a pregnant mother, they may have adverse effects when ingested by someone for whom they were not intended. Recent research has suggested that exposure to estrogens in milk may be involved with several different human health issues, including cancer and infertility (Ganmaa et al., 2001; Qin et al., 2004; Ganmaa and Sato, 2005).

Although several studies have measured estrogens in milk, many conclude that the levels of estrogens in cow milk are too low to pose a risk to human health (Monk et al., 1975; Wolford and Argoudelis, 1979; Hartmann et al., 1998; Pape-Zambito et al., 2007). Further, data show that commercial processing can decrease the quantity of estradiol present in milk (Wolford and Argoudelis, 1979). However, low-fat (1\%) cow milk was recently found to have significant estrogenic activity in terms of its effects on the rat reproductive tract (Ganmaa et al., 2006).

The female rat is a useful model for determining estrogenic activity. It is well established that an in- 
crease in uterine wet weight and keratinization of the vaginal epithelium occurs following treatment with an estrogen or an estrogen mimic (Lauson et al., 1939). In the nervous system, estrogen can also act within the hippocampus, to decrease anxiety in mammals (Walf and Frye, 2007).

Therefore, if commercial cow milk contained biologically active estrogens, it could be predicted that treatment of the ovariectomized female rat would increase uterine weight and also decrease anxiety levels. Further, estrogens are lipophilic and studies of cow milk have found the concentrations of fat in the milk to be positively correlated to the amount of estrogens measured within the milk (Hartmann et al., 1998; Pape-Zambito et al., 2007, 2008). Therefore, it could be hypothesized that milk with greater fat content would have a greater uterotrophic and anxiolytic effects than milk with less fat. This study was performed to examine whether biologically active estrogens are present in commercial milk as well as if estrogen content differs by fat content of milk.

\section{MATERIALS AND METHODS}

\section{Animals}

Female Sprague-Dawley rats were produced on site from the breeding colony at Framingham State University (Framingham, MA). At weaning age, females were housed 2 per cage. The cages were made of plastic (33 $\times 20 \times 19 \mathrm{~cm})$ with wire lids, which allowed ad libitum access to rat chow and water. Animals were kept in a reverse light room, with lights on at $2200 \mathrm{~h}$ and lights off at $1100 \mathrm{~h}$, which allowed the rats to be active during the daytime. All animal care and procedures were approved by the Framingham State University Institutional Animal Care and Use Committee.

\section{Surgery}

At about 8 wk of age, after the rats had reached puberty, animals underwent a bilateral ovariectomy (to remove the sources of endogenous estrogens). The rats were first anesthetized with $75 \mathrm{mg} / \mathrm{kg}$ of ketamine (Butler Schein Animal Health, Dublin, OH) and 2.5 $\mathrm{mg} / \mathrm{kg}$ of acepromazine (i.p.; Butler Schein Animal Health). Once the animal was unconscious, a small skin incision was made between the ribs and the pelvis on the side of the rat. A muscle incision was then made so the fat pads containing the ovaries were found underneath. The ovaries were extracted by pulling out the periovarian fat through the muscle incisions. A ligature was secured around the fallopian tube and uterine horn to prevent bleeding. A cut was then made distal to the uterine horn. The horn was then put back into the abdominal cavity and the muscle wall was sutured. The skin incision was then closed with surgical staples. The rats were given $15 \mathrm{mg}$ of ibuprofen $/ \mathrm{kg}$ orally for pain control and were monitored until they were fully conscious and checked for any excess bleeding. The rats were allowed $7 \mathrm{~d}$ to recover from surgery before experimental treatment began. After surgery, each animal was housed individually for the remainder of the experiment.

Sham surgeries were performed for rats in the procedural control group to control for any trauma related to the surgical procedure. The procedure was identical to the ovariectomy procedure; however, instead of the ligation and removal of the ovaries, the ovaries were put back into the abdominal cavity and the incision was closed with surgical staples. The rats were also given ibuprofen for pain control and were monitored until they were fully conscious and checked for any excess bleeding. These rats were also allowed $7 \mathrm{~d}$ to recover from surgery before experimental treatment began. After surgery, each animal was housed individually for the remainder of the study. Both the ovariectomized and the sham ovariectomized animals underwent a daily vaginal smear beginning the first day of the treatment to examine the vaginal epithelium for evidence of estrogen exposure (Jones and Edgren, 1973).

\section{Treatment}

The experimental rats were randomly divided into 4 groups (7 rats per group) and each group was given a different type of local commercially available milk: skim, $1 \%, 2 \%$, and whole milk to assess if the fat content was correlated with the estrogenic quality of the milk. The treatment regimen for the ovariectomized animals consisted of $14 \mathrm{~d}$ in which the rats were given $150 \mathrm{~mL}$ of milk, as well as water ad libitum, each day. The volume of the milk consumed was recorded each day to determine whether a significant difference in milk consumption existed between groups. The amount of water consumed was not determined.

Two control groups were used in this experiment (7 rats per group). The negative control group consisted of ovariectomized rats that were given only water (no milk) during the treatment period. The procedural control group consisted of rats that underwent a sham surgery to control for anesthesia and surgical trauma, but ovaries were left in place. These animals were given water instead of milk during the treatment period.

All of the animals that were ovariectomized underwent behavioral testing on treatment d 12 and 13 and were killed on treatment d 15 (24 h after the last milk treatment). However, due to the cyclical nature of the 
rodent reproductive system, this same timeline was not possible with the sham control animals. These animals underwent a vaginal smear every day, to determine whether they were in proestrus or estrus. Behavioral tests were performed on 2 sequential days, beginning with the first proestrus smear closest to d 12 of treatment. Immediately following the conclusion of the second behavioral test, these animals were anesthetized and their uteri were collected. This variation from the ovariectomized protocol was required to ensure that these animals were tested and collected with high circulating estrogen level, and therefore were appropriate estrogen-exposed controls.

\section{Behavioral Tests}

Two separate tests were used to examine anxiety levels: elevated plus maze and open field. Animals that have higher levels of circulating estrogen have lower anxiety levels (Walf and Frye, 2007). The person administering the test was blind to the treatment group to which the animal belonged. The behavioral tests were administered between 1400 and $2000 \mathrm{~h}$, under minimal room light.

The first behavioral test the rats were exposed to was the elevated plus maze, which consisted of 4 arms: 2 open with lengths of $50.8 \mathrm{~cm}$ and widths of $11.43 \mathrm{~cm}$, and 2 arms enclosed by walls $30.5 \mathrm{~cm}$ tall $(50.8 \times 11.43$ $\times 30.5 \mathrm{~cm})$. In the center was an open section $(10.16$ $\times 10.16 \mathrm{~cm}$ ) where the rat was initially placed and its behavior was observed for $5 \mathrm{~min}$ in terms of time spent in the open arms or closed arms and the number of transitions made between them. A rat that spent more time in the open arms or made a larger number

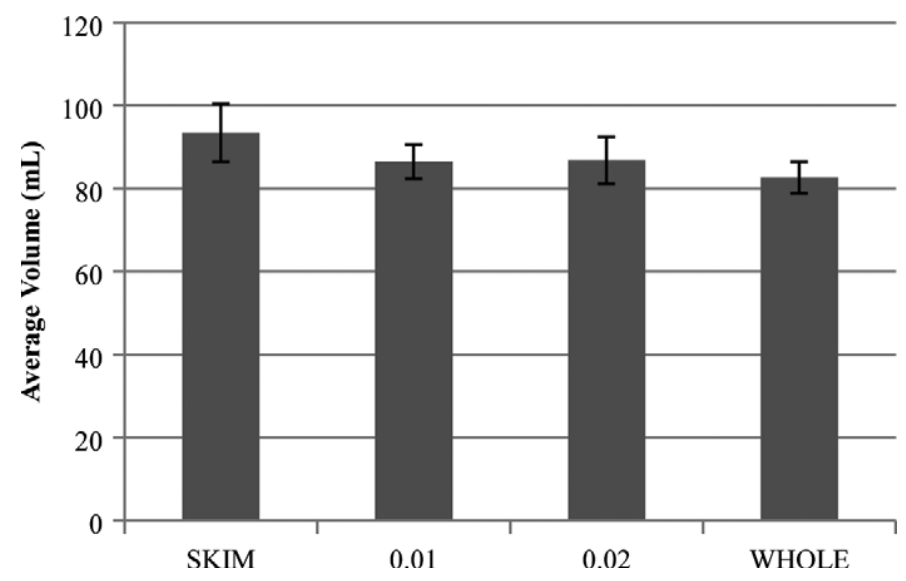

Figure 1. Average daily volume of milk consumed per rat per group (groups received skim, $1 \%, 2 \%$, or whole milk). No difference was observed in the average volume of milk consumed daily between any of the experimental groups (ANOVA, $P=0.55$ ). of transitions was considered to have less anxiety and, therefore, greater levels of circulating estrogen.

The second behavioral test the rats underwent was an open field test. This test was done in a transparent plastic box $(122 \times 86.4 \times 30.5 \mathrm{~cm})$. The bottom of the box had been divided into a visible grid of $17.8 \times 19-\mathrm{cm}$ boxes. A $40-\mathrm{W}$ bulb hung over the middle of the box, where the rat was initially placed, and her behavior was observed for $5 \mathrm{~min}$ in terms of the number of inner crosses made (crosses made on inner lines of the box) versus the number of outer crosses made (crosses made around the lines on the outer rim of the box). A rat that made a greater number of inner crosses or had more exposure to the light was considered to have less anxiety and, therefore, greater levels of circulating estrogen.

\section{Uterotrophic Assay}

Each rat was euthanized via carbon dioxide inhalation. The body cavity was opened and the uterus was removed, and then trimmed of all fat. Next, each uterus was blotted with filter paper to remove excess fluids and weighed.

\section{Statistical Analysis}

All data were analyzed by a 1-way ANOVA. A Tukey post-hoc test was administered, when necessary, to determine what differences existed among groups. Tests were considered significant for all $P<0.05$. Results are given as means \pm standard deviation.

\section{RESULTS}

The average volume of milk consumed daily ranged from 82.7 to $93.5 \mathrm{~mL} /$ animal and was not statistically different across all 4 milk groups (ANOVA, $P=0.554$; Figure 1).

For the elevated plus maze, the sham ovariectomized animals, who received no milk, spent a significantly greater amount of time in open arms compared with all other groups (ANOVA, $P<0.001$, Tukey post-hoc test; Figure 2). These results are typical of those expected of gonadally intact animals with high levels of circulating estrogen, whether or not the animals underwent the sham surgery. No significant difference was found between the negative control animals and the other milk groups and no significant difference was found among the different milk groups for the amount of time spent in open arms. Also, no significant difference was found between groups for the total number of transitions made in the elevated plus maze (ANOVA, $P=0.209$; data not shown). 


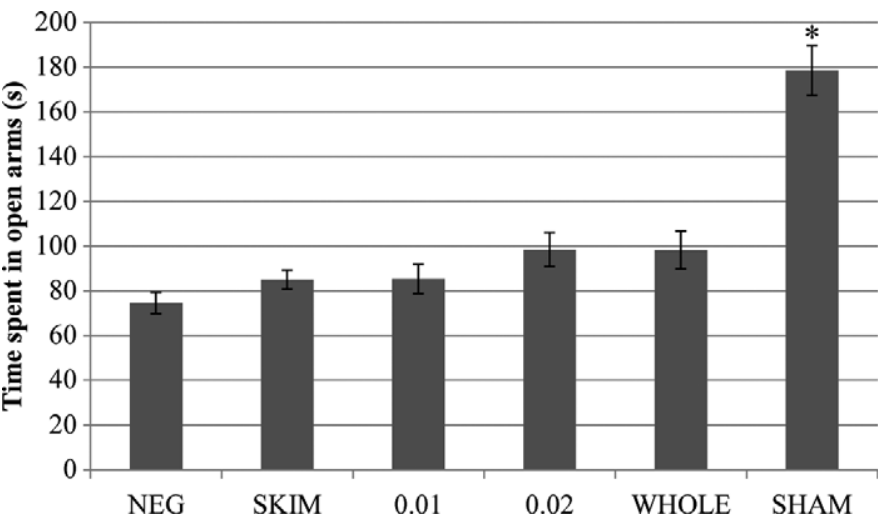

Figure 2. Elevated plus maze behavioral test for estrogens. The sham animals spent more time in the open arms compared with the negative control animals as well as any of the animals that were treated with milk (ANOVA, $P<0.001$ ). No difference was observed between the negative control (NEG) animals and any of the milk groups (that received skim, $1 \%, 2 \%$, or whole milk). Also, no difference existed between any of the milk groups. The sham ovariectomized animals were tested with high endogenous circulating estrogen levels.

For the open field test, the sham ovariectomized animals, who received no milk, made a significantly greater number of inner crosses compared with all other groups (ANOVA, $P<0.001$; Tukey post-hoc test; Figure 3). Again, these data are similar to those expected from animals that did not undergo surgery. No significant difference was found between the negative control animals and the other milk groups. Also, no significant difference was found among the different milk groups for number of inner crosses made. The sham ovariectomized animals also made a significantly greater number of total crosses compared with the all other groups (ANOVA, $P<0.001$; data not shown).

Uterine weight was significantly greater in the sham ovariectomized animals when compared with any other group (ANOVA, $P<0.05$; Table 1 ). The average uterine weight for this group was $418 \pm 144 \mathrm{mg}$, whereas all the other groups had average uterine weights of only about one-fourth to one-third of that. No significant differences were observed between the negative control and any of the milk groups. Also, no differences existed in uterine weights among any of the milk groups.

\section{DISCUSSION}

There is growing concern of whether or not exposure to the estrogens from milk can cause adverse effects in the consumer. Qin et al. (2004) have demonstrated that estrogens from milk can cause the growth of mammary tumors in rats and ongoing investigations by Ganmaa and Sato (2005) are evaluating the possibility of a positive correlation between the rise in certain types of hormone-related cancer levels in humans and estrogens

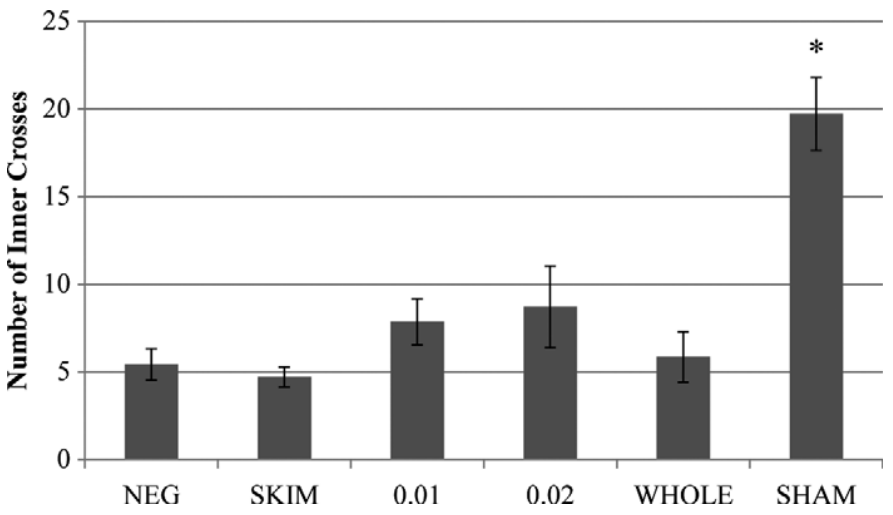

Figure 3. Open field test for physiologically active estrogen. The sham animals exhibited a greater number of inner crosses compared with the negative control animals and all of the animals that were treated with milk (ANOVA, $P<0.001$ ). No difference was observed between the negative control animals (NEG) and any of the milk groups (that received skim, $1 \%, 2 \%$, or whole milk). Also, no difference existed between any of the milk groups. The sham ovariectomized animals were tested when circulating estrogen levels were high.

found in cow milk. Therefore, it is vital that the public be made aware of the possible biological effects that the consumption of milk may have had.

The objective of this experiment was to determine if commercial cow milk exhibits estrogenic effects in the rat. Also, this study addressed whether any correlation existed between fat content of the milk and an estrogenic effect. Previous studies using the rat model have shown estrogenic effects of cow milk on the rat uterus (Ganmaa et al., 2004, 2006), but this is the first study to also examine possible effects on the nervous system. Estrogens, when bound to estrogen receptors in the hippocampus, have been known to result in anxiolytic effects in mammals (Walf and Frye, 2007).

The data collected in this experiment do not support the conclusion that significant amounts of biologically active estrogens are present in cow milk or that a differ-

Table 1. Uterine wet weights after $14 \mathrm{~d}$ of treatment ${ }^{1}$

\begin{tabular}{lcc}
\hline Group & $\begin{array}{c}\text { Average uterine } \\
\text { weight }(\mathrm{mg})\end{array}$ & SD \\
\hline Negative control & 113 & 16.16 \\
Skim & 123 & 13.15 \\
0.01 & 121 & 11.34 \\
0.02 & 125 & 28.29 \\
Whole & 151 & 61.14 \\
Sham & $418^{*}$ & 144.84 \\
\hline
\end{tabular}

${ }^{1}$ The sham ovariectomized animals exhibited a greater uterine weight compared with the negative control animals and all of the animals that were treated with milk ( ${ }^{*}=$ ANOVA, $P<0.001$ ). No difference was observed between the negative control animals and any of the milk groups (that received skim, $1 \%, 2 \%$, or whole milk). Also, no difference existed between any of the milk groups. The sham animals were collected when circulating estrogen levels were high. 
ence in estrogen content exists between milk with different fat content. The overall volume of milk consumption was not a confounding factor in the analysis, as it was determined that no significant difference existed for milk consumption between groups.

The results from the elevated plus maze and the open field test do not support the initial hypothesis that milk consumption would have an anxiolytic effect on rats, as no significant difference was found between the milk groups and the negative control group. Also, the results do not support the second hypothesis that increasing the fat content of the milk would have had an increasing anxiolytic effect on the rats, as no significant difference was found among the different milk groups for all analyses of behavior across those 2 tests. These data support the conclusion that a sufficient amount of estrogens within milk does not exist to manifest a behavioral effect.

The uterine wet weight data further support the conclusions of the behavioral studies that there are not significant levels of biologically active estrogens in commercial milk, regardless of fat content. Drinking milk, regardless of fat content, did not result in a uterotrophic effect any greater than drinking tap water. The sham ovariectomized group, which contained intact ovaries had significantly heavier uteri, confirming the uterotrophic effect of endogenous estrogens. It is interesting to note the difference in variation in uterine weight between the animals that consumed the different fat content groups, which ranged from 9\% (1\% milk group) to $40 \%$ (whole milk group). However, even with this high variability, the mean uterine weight of the shamovariectomized group is over 2.5 times greater than the mean uterine weight of the whole milk group, supporting the conclusion of a lack of biologically active estrogens in the milk. Further, we have also examined for uterotrophic effects using unprocessed organic whole milk from pasture-fed cows from another local vendor, and found no effect when compared with animals that consumed tap water (D. Maroun and B. W. Snyder, unpublished data).

Recently, several studies have reported addressing the issue of whether or not biologically active estrogens are present in cow milk when consumed orally. Unfortunately, due to lack of consistent experimental design, supplies, animals, and procedures, it is difficult to make any broad conclusions regarding this topic. The current study was designed in a similar manner to those previously published by Ganmaa et al. (2004, 2006); however, there were some notable differences. For example, animals in this study were fed milk along with chow pellets, whereas the Ganmaa et al. (2004, 2006) studies used a chow powder that was mixed with milk or artificial milk. However, previous investigations have shown no estrogenic effects on behavior when the pellet chow was compared with a low-soy rat chow (A. M. Davis, unpublished data). Therefore, it is unlikely that the food source is a candidate for the difference. The current study examined the effects over a 2-wk treatment period, whereas the Ganmaa et al. (2006) study used a 7-d treatment period. However, the Ganmaa et al. (2004) study used a treatment that lasted over 10 wk (Ganmaa et al., 2004). Interestingly the increase in uterine weight was found in the 7-d exposure, whereas the longer exposure resulted in no observable problems with reproductive organ development or fertility (Ganmaa, et al., 2004, 2006). Also, the type of rat was not the same in each study. The Ganmaa et al. (2004, 2006) studies used Wistar GALAS Hannover rats, whereas the current study used Sprague-Dawley rats.

Other reports have been published on estrogenic activity in milk (Zhou et al., 2010). Zhou et al. (2010) also used Sprague-Dawley rats; however, they used ovariectomized, immature rats. These animals were exposed to powdered chow and milk for $7 \mathrm{~d}$ and used a negative control group that received powdered chow and water. The uterine weights reported in the Zhou et al. (2010) study were slightly lower than those reported here. However, given the smaller, younger rat model used, it is not unexpected. Unfortunately, no estrogentreated group was included in the Zhou et al. (2010) study.

A study by Nielsen et al. (2009) also examined the possible estrogenic effects of milk, using both in vitro and in vivo techniques. The in vivo portion of this study used a gonadally intact immature mouse. The animals in this study were provided animal chow and milk for 7 d. Results show no difference in uterine weights between animals that were treated with milk or water (Nielsen et al., 2009). However, animals that were treated orally with water containing estradiol had 3 to 4 times the uterine weight of the animals that had consumed either milk or water (Nielsen et al., 2009), which is similar to our results for the sham ovariectomized females. It is interesting to note that, in their study, the milk used was able to activate the estrogen receptor in an in vitro assay (Nielsen, et al., 2009). These data suggest that milk processing by the digestive tract may be important to making it inactive, in terms of estrogen activity.

The final possible variable is the milk supply. The milk in the Ganmaa et al. $(2004,2006)$ studies was produced locally in Japan, the Zhou et al. (2010) study used milk from China, the Nielsen et al. (2009) study used milk from Denmark, and the current study used milk produced in the United States. It is unknown whether differences exist in the milking practices, animal care, animal feeding, and milk preparation between the different countries, and whether these differences, 
if any, could be responsible for the differences in results. However, given the variability in estrogen levels reported in milk from cows around the United States (Monk et al., 1975; Wolford and Argoudelis, 1979; Pape-Zambito et al., 2007), one can only speculate that similar differences would be found around the world. Until these differences in care and preparation can be made uniform, it may be impossible to make a sweeping conclusion regarding the content of biologically active estrogens in commercial milk.

Even though estrogens typically cause body changes to occur in a matter of hours or days, some future studies may wish to examine if a prolonged consumption of milk could have an estrogenic effect on the consumers by performing this same experimental design on rats for an extended period of time. This may be significant, as humans often consume milk products over a long period of time, or throughout most of their lives, and prolonged exposure to milk may have additive effects that could result in adverse effects on the consumer. However, given the current study, we conclude that no biologically active estrogens are present in the commercial milk produced in the manner of our samples, regardless of fat content. Further, because the milk products used in this study were not found to be estrogenic, these milk products do not appear to pose any risk on its consumers for estrogen-enhanced tumor growth or other estrogen related health issues.

\section{ACKNOWLEDGMENTS}

This study was sponsored by the Biology Department and the Center for Excellence in Learning, Teaching, Scholarship and Service at Framingham State University (Framingham, MA).

\section{REFERENCES}

Cunningham, K. D., M. J. Cecava, T. R. Johnson, and P. A. Ludden. 1996. Influence of source and amount of dietary protein on milk yield by cows in early lactation. J. Dairy Sci. 79:620-630.

Erb, R. E., M. M. Goodwin, R. A. Morrison, and A. O. Shaw. 1952. Lactation studies. I. Effect of gestation. J. Dairy Sci. 35:224-233.

Erb, R. E., B. P. Chew, and H. F. Keller. 1977. Relative concentration of estrogen and progesterone in milk and blood and excretion of estrogen in urine. J. Anim. Sci. 45:617-626.
Ganmaa, D., L.-Q. Qin, P.-Y. Wang, H. Tezuka, S. Teramoto, and A. Sato. 2004. A two-generation reproduction study to assess the effects of cows' milk on reproductive development in male and female rats. Fertil. Steril. 82:1106-1114.

Ganmaa, D., and A. Sato. 2005. The possible role of female sex hormones in milk from pregnant cows in the development of breast, ovarian and corpus uteri cancers. Med. Hypotheses 65:1028-1037.

Ganmaa, D., H. Tezuka, D. Enkhmaa, K. Hoshi, and A. Sato. 2006. Commercial cows' milk has uterotrophic activity on the uteri of young ovariectomized rats and immature rats. Int. J. Cancer 118:2363-2365.

Ganmaa, D., P. Y. Wang, L. Q. Qin, K. Hoshi, and A. Sato. 2001. Is milk responsible for male reproductive disorders? Med. Hypotheses 57:510-514.

Gowen, J. W. 1924. Intrauterine development of the bovine fetus in relation to milk yield in Guernsey cattle. J. Dairy Sci. 7:311-317.

Hartmann, S., M. Lacorn, and H. Steinhart. 1998. Natural occurrence of steroid hormones in food. Food Chem. 62:7-20.

Heap, R. B., and M. Hamon. 1979. Oestrone sulphate in milk as an indicator of a viable conceptus in cows. Br. Vet. J. 135:355-363.

Jones, R. C, and R. A Edgren.. 1973. The effect of various steroids on the vaginal histology in the rat. Fertil. Steril. 24:284-291.

Komaragiri, M. V. S., and R. A. Erdman. 1997. Factors affecting body tissue mobilization in early lactation dairy cows: Effect of dietary protein on mobilization of body fat and protein. J. Dairy Sci. 80:929-937.

Lauson, H. D., C. G. Heller, J. B. Golden, and E. L. Sevringhaus. 1939. The immature rat uterus in the assay of estrogenic substances, and a comparison of estradiol, estrone and estriol. Endocrinology 24:35-44.

Monk, E. L., R. E. Erb, and T. A. Mollett. 1975. Relationships between immunoreactive estrone and estradiol in milk, blood, and urine of dairy cows. J. Dairy Sci. 58:34-40.

Nielsen, T. S., J. V. Nørgaard, S. Purup, X. C. Fretté, and E. C. Bonefeld-Jørgensen. 2009. Estrogenic activity of bovine milk high or low in equol using immature mouse uterotrophic responses and an estrogen receptor transactivation assay. Cancer Epidemiol. 33:61-68.

Pape-Zambito, D. A., A. L. Magliaro, and R. S. Kensinger. 2007. Concentrations of $17 \beta$-estradiol in Holstein whole milk. J. Dairy Sci. 90:3308-3313.

Pape-Zambito, D. A., A. L. Magliaro, and R. S. Kensinger. 2008. $17 \beta$-estradiol and estrone concentrations in plasma and milk during bovine pregnancy. J. Dairy Sci. 91:127-135.

Qin, L.-Q., J.-Y. Xu, P.-Y. Wang, D. Ganmaa, J. Li, J. Wang, T. Kaneko, K. Hoshi, T. Shirai, and A. Sato. 2004. Low-fat milk promotes the development of 7,12-dimethylbenz(a)anthracene (DMBA)-induced mammary tumors in rats. Int. J. Cancer 110:491-496.

Walf, A. A., and C. A. Frye. 2007. Administration of estrogen receptor beta-specific selective estrogen receptor modulators to the hippocampus decrease anxiety and depressive behavior of ovariectomized rats. Pharmacol. Biochem. Behav. 86:407-414.

Wolford, S. T., and C. J. Argoudelis. 1979. Measurement of estrogens in cow's milk, human milk, and dairy products. J. Dairy Sci. 62:1458-1463.

Zhou, H., L.-Q. Qin, D.-F. Ma, Y. Wang, and P.-Y. Wang. 2010 Uterotrophic effects of cow milk in immature ovariectomized Sprague-Dawley rats. Environ. Health Prev. Med. 15:162-168. 